\title{
CLOSED FORM SURFACE RAY TRACING ON OGIVAL SURFACES
}

RM Jha, SA Bokhari, $V$ Sudhakar and PR Mahapatra

Department of Aerospace Engineering

Indian Institute of Science

Bangalore, 560012 India

\section{ABSTRACT}

A new method based on the geodesic constant has been developed by the authors for the surface ray tracing on an ogive. The surface ray geometric parameters required in the high frequency EM calculations are obtained in explicit analytical form. The ogive is treated as a coordinate surface of the bispherical coordinate system which is a geodesic coordinate system. All the ray geometric parameters are obtained in one-parameter form in terms of the First Geodesic Constant h. These ray parameters may be used in the antenna radiation pattern, mutual coupling and radar cross section calculations.

\section{Introduction}

The importance of the ogival surfaces lies in the fact that they, along with the Eisenhart (quadric) cylinders and surfaces of revolution, describe nearly all the gross external shapes encountered in Aerospace Engineering [1]. The Eisenhart quadric cylinders and surfaces of revolution are easily described in the well known eleven second-degree coordinate systems referred to as the Eisenhart Coordinate System [2]. In contrast, an ogive is not a coordinate surface of an Eisenhart Coordinate system and cannot be readily parametrized as such.

In this paper, an ogive is identified as the coordinate surface of a NonEisenhart Coordinate System, namely the Bispherical Coordinate system. A surface ray analysis is carried out and all the ray geometric parameters required for the high frequency EM antenna analysis have been obtained in oneparameter form, for the first time in open literature.

\section{Formulation}

The parametric equation of an ogive in Bispherical Coordinate System is given as [3]

$$
x=\frac{R \sin a \cos \phi}{\cosh n-\cos a} \quad y=\frac{R \sin a \sin \phi}{\cosh n-\cos a} \quad z=\frac{R \sinh n}{\cosh n-\cos a} \quad a>\pi / 2
$$

where $\mathrm{a}$ and $\mathrm{R}$ are the shaping parameters of the ogive. We identify the coordinates $(n, \phi)$ with the geodesic coordinates $(u, v)$ so that eq $(1)$ is of the form

$$
x=f(u) \cos v \quad y=f(u) \sin v \quad z=g(u)
$$


where

$f(u)=\frac{R \sin a}{\cosh u-\cos a} \quad g(u)=\frac{R \sinh u}{\cosh u-\cos a}$

and the first and second partial derivatives of $f(u)$ and $g(u)$ are obtained as

$f_{u}=\frac{R \sinh u \sin a}{(\cosh u-\cos a)^{2}}$
$f_{u u}=\frac{R \sin a\left(1-\sinh ^{2} u-\cosh u \cos a\right)}{(\cosh u-\cos a)^{3}} g_{u u}=\frac{R \sinh u(\cosh u \cos a-1)}{\left.(\cosh u-\cos a)^{2} a-\cosh u \cos a\right)}$
$(\cosh u-\cos a)^{3}$

The subscript u denotes the partial derivatives w.r.t. u. It has been shown by the authors that the Bispherical Coordinate System is an example of a Geodesic Coordinate System [3]. Hence the general ray analysis developed by these authors for surface ray analysis in the Geodesic Coordinate System can be extended to the ogive as well. All the surface ray geometric parameters (of the differential form) for the ogive can now be readily obtained by merely substituting the value of $f_{u}, g_{u}, f_{u u}$ and $g_{u u}$ in the appropriate equations. In particular, the right (i.e. anticlockwise) $\mathrm{m}$-th order geodesic, which completely encircles the ogive $\mathrm{m}-\mathrm{l}$ times is expressed as [3]

$$
\frac{d v}{d u}=\frac{t h m\left(f_{u}{ }^{2}+g_{u}{ }^{2}\right)^{1 / 2}}{f(u)\left[f(u)^{2}+h_{m}^{2}\right]^{1 / 2}}
$$

where $h_{r m}$ is the First Geodesic Constant obtained by integrating of the second order canonical differential equation of the geodesic. The positive (negative) sign of $h_{r m}$ depends on the increasing (decreasing) nature of $v$ parameter, which in general, is of the form

$$
v=v\left(u, h_{m n}\right)+h_{m n}^{\prime}
$$

$h_{r m}^{\prime}$ is the Second Geodesic Constant of the geodesic. $h_{r m}$ and $h_{r m}^{\prime}$ can be determined since the source and observation point coordinates are known a priori. All the surface ray geometric parameters required for the high frequency methods are expressed analytically in terms of $h_{r m}$. For example, the rectangular coordinate components of the unit tangent vector $\hat{t}$ are expressed as

$$
\hat{t}=t_{x} \hat{i}+t_{y} \hat{j}+t_{z} \hat{k}
$$

where

$$
\begin{aligned}
& t_{x}=\frac{f_{u}\left[f(u)^{2}-h_{m}^{2}\right]^{1 / 2} \cos v-\left(f_{u}^{2}+g_{u}^{2}\right){ }^{1 / 2} h_{m} \sin v}{f(u)\left(f_{u}^{2}+g_{u}^{2}\right)^{1 / 2}} \\
& t_{y}=\frac{f_{u}\left[f(u)^{2}-h_{m m}^{2}\right]^{1 / 2} \sin v-\left(f_{u}^{2}+g_{u}^{2}\right)^{1 / 2} h_{m} \cos v}{f(u)\left(f_{u}^{2}+g_{u}^{2}\right)^{1 / 2}}
\end{aligned}
$$




$$
t_{z}=\frac{g_{u}\left[f(u)^{2}-h_{m m}^{2}\right]^{1 / 2}}{f(u)\left(f_{u}^{2}+g_{u}^{2}\right)^{1 / 2}}
$$

Similarly $\hat{n}$ and $\hat{b}$ of the Frenet-frame field vectors can be readily derived. We summarily present the expression for some of the key ray geometric parameters (on the ogive) required in the ray-theoretic formulation. The radius of curvature $\rho_{g}(u)$ and torsion $\tau(u)$ are readily obtained as

$$
\begin{aligned}
& \rho_{g}(u)=\frac{f^{3}\left(f_{u}^{2}+g_{u}^{2}\right)^{3 / 2}}{-f^{3} g_{u^{f} u u^{+}}{ }^{3} f_{u} g_{u u}+h_{m}^{2} f_{u_{u}}^{f} u u^{-} h_{m m}^{2} f f_{u} g_{u u}+h_{m m}^{2} f^{2} g_{u}+h_{m m}^{2} g_{u}^{3}} \\
& \tau(u)=\frac{-f_{u_{u}} u_{u}+f f_{u} g_{u u}-f^{2} g_{u}-g_{u}^{3}}{f^{3}\left(f_{u}^{2}+g_{u}^{2}\right)^{3 / 2}}
\end{aligned}
$$

The arc length $s_{r m}$ of the right $m$-th order geodesic is obtained by integrating the metric

$$
s_{m m}=\int_{S}^{P_{\lambda m}} \frac{h_{m n}\left(f_{u}^{2}+g_{u}^{2}\right)^{1 / 2}}{f(u)\left[f(u)^{2}-h_{m}^{2}\right]^{1 / 2}} d u
$$

The generalized Fock parameter $\boldsymbol{\xi}_{\mathrm{rm}}$ is defined as the integral of a function of the radius of curvature $\rho_{g}$ along the geodesic with respect to the metric, from $\mathrm{S}$ to $\mathrm{P}_{\mathrm{rm}}$

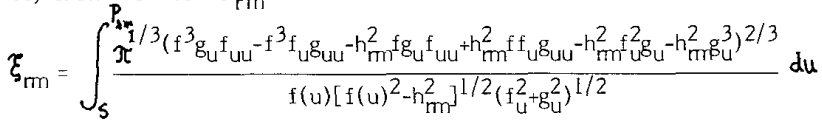

The generalized Fock parameter $\boldsymbol{\xi}_{\mathrm{rm}}$ is used in computing the generalized Fock integrals $\tilde{U}\left(\boldsymbol{\xi}_{\mathrm{rm}}\right)$ and $\tilde{V}\left(\boldsymbol{\xi}_{\mathrm{rm}}\right)$, which is the crux of UTD computation [4].

\section{Discussion}

The use of Eisenhart Coordinate System has enabled the EM analysis on a very wide class of commonly used quadric surfaces, namely the Eisenhar surfaces, which are extremely important from the surface modeling point of view. However, there are surfaces, equally important from structural considerations in aerospace engineering which are not Eisenhart surfaces. Ogive is one such rotationally symmetric Non-Eisenhart surface where surface ray analysis until now has been extremely difficult.

All the ray geometric parameters required in the high frequency analysis has been obtained in this paper by a Geodesic Constant Method (GCM) developed by the authors. Furthermore, eq (2) implies that the ray parameters obtained here are applicable to the complete class of rotationally symmetric Non-Eisenhart surfaces, in general. The demonstrable ease of its application to the surface analysis for relatively complex surfaces shows the power of the GCM. 


\section{REFERENCES}

[1] R.M. Tha: "Surface Ray Tracing on Convex Quadrics with Applications to Analysis of Antennas on Complex Aerospace Bodies", 39th International Astronautical Congress of the International Astronautical Federation, Bangalore, India, Oct. 8-15, 1988.

[2] P. Moon and D.E. Spencer, Field Theory Handbook. Bertin: Springer-Verlag, 1971.

[3] R.M. Tha, Surface Ray Tracing on Convex Quadrics with Applications to Mutual Coupling between Antennas on Aerospace Bodies, Ph D Dissertation. Submitted to Department of Aerospace Engineering, Indian Institute of Science, Bangalore, India, Nov. 1988.

[4] P.H. Pathak and N. Wang, "Ray analysis of mutual coupling between antennas on a convex surface", IEEE Trans. Antennas \& Propagat. (USA), vol. AP-29, no. 6, pp. 911-922, Nov. 1981 .

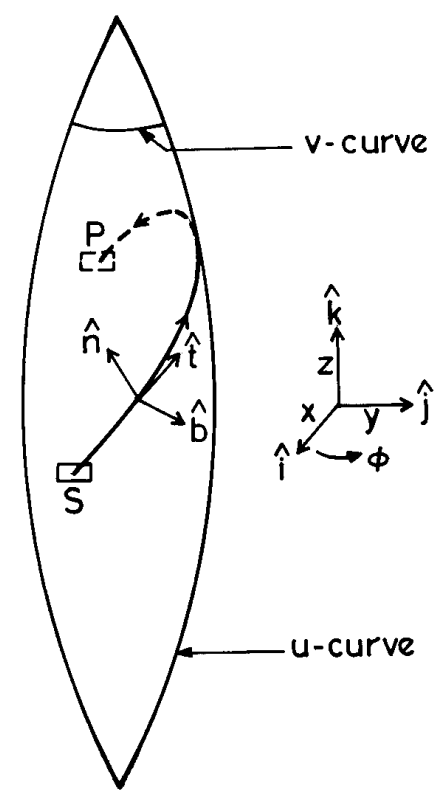

Fig. 1 Ray analysis on a circular ogive. 